\title{
Self-reporting styrylthiazolium photopharmaceuticals: mitochondrial localisation as well as SAR drive biological activity
}

\author{
Li Gao, Yvonne Kraus, Andrea Stegner, Thomas Wein, Constanze Heise, Leonie von Brunn, \\ Elena Fajardo-Ruiz, Julia Thorn-Seshold, Oliver Thorn-Seshold*
}

Department of Pharmacy, Ludwig-Maximilians-Universität, München, 5-13 Butenandtstrasse, München 81377, Germany.

ORCID: OT-S: 0000-0003-3981-651X

*correspondence to: oliver.thorn-seshold@cup.Imu.de

Keywords: microtubules, mitochondria, photopharmacology, combretastatin, delocalized lipophilic cations

\begin{abstract}
Novel photoswitches with features complementary to the well-established azobenzenes are increasingly driving high-precision research in photopharmacology in cells and in vivo. Styrylthiazolium (StyTz) and styrylbenzothiazolium (StyBtz) are cellularly untested E/Z-isomerisation photoswitches which are nearly isosteric to azobenzenes, but have distinct photophysical and physicochemical properties: including ca. $60 \mathrm{~nm}$ red-shifted $\Pi \rightarrow \pi^{*}$ absorption, self-reporting fluorescence, $Z \rightarrow E$ relaxation that matches typical biological timescales, and good solubility due to their permanent positive charge. We here tested StyTz and StyBtz for their potential as photopharmaceutical scaffolds, by applying them to photocontrol the dynamics of the microtubule cytoskeleton. We observed light-specific disruption of microtubule network architecture and antiproliferative activity, with a structure-activity relationship matching expectations for tubulin inhibitors. However, while testing the lead StyBtz2 for its molecular mechanism of action, we found that it did not inhibit microtubule dynamics. We tracked its localisation in live cells by relying on its self-reporting fluorescence, observing accumulation of $E$-StyBtz2 into mitochondria; after several minutes of illumination it was then released into the cytosol concomitant with blebbing and cell death. We interpret this as light-dependent catastrophic rupturing of mitochondria on acute timescales. We conclude that StyTz/StyBtz can be interesting photopharmaceutical scaffolds for addressing mitochondrial, rather than cytosolic, targets.
\end{abstract}




\section{Introduction}

Photoswitchable small molecules are powerful tools for high-precision manipulations of a range of biological processes in live animals (in vivo) or in live cells. ${ }^{1,2}$ They may even be irreplaceable as tools to properly address systems in which spatiotemporal patterning of biological activity on the subcellular/cellular or tissue level is crucial: e.g. microtubule and actin dynamics in cytoskeleton-supported motion (subcellular/cellular level) $)^{3,4}$, or firing of retinal neurons for vision restoration (tissue level) ${ }^{5,6}$.

Hartley first characterised azobenzene as an $E / Z$ photoswitch in $1937^{7}$. After Erlanger's pioneering applications in protein and neuron control, ${ }^{8,9}$ azobenzenes became the most widely-used photoswitch from materials sciences ${ }^{10}$ to chemical biology. ${ }^{2,11}$ Recently though, a range of other photoswitches with distinct features are emerging, for better photocontrol of specific biological systems ${ }^{12,13}$. Often, these are differentiated in their kinetics of relaxation to the thermodynamically more stable isomer, ${ }^{14}$ and on shifting their photoisomerisation "action spectra": either to the near-UV, to avoid photoresponse during imaging while permitting photoactivation with the common $405 \mathrm{~nm}$ laser; ${ }^{15}$ to the mid-visible, for efficient patterning with imaging laser lines $;{ }^{16}$ or towards the red, for photoresponse to light that penetrates more deeply through complex environments such as tissues. ${ }^{17}$

Here, we desired to explore scaffolds that are as-yet untested for cellular photopharmacology, but which offer a desirable balance of properties that neither azobenzenes nor other photoswitches such as heterostilbenes provide: chiefly, efficient $405 \mathrm{~nm}$ excitation, no photoresponse to imaging lines, self-reporting fluorescence, good solubility, and relaxation on biologically relevant timescales. We furthermore aimed at test them in a proof of concept biological application: photocontrolling the functionality of the microtubule cytoskeleton.<smiles>[X]c1ccc(OC)c(OC)c1</smiles>

CA4: $\mathrm{X}=\mathrm{CH}$

Z-PST-1: $X=N$

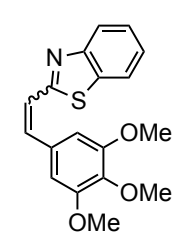

SBTub2 north ring replaced

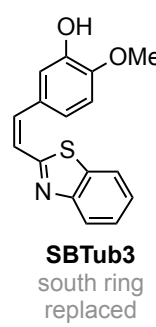

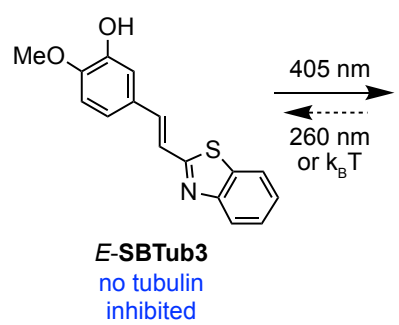

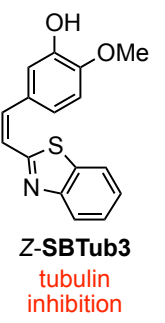

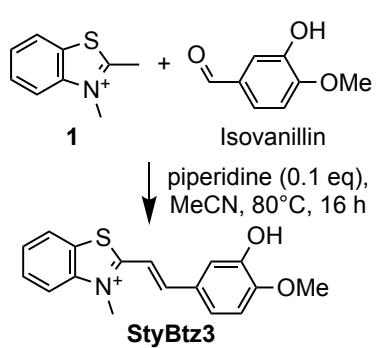
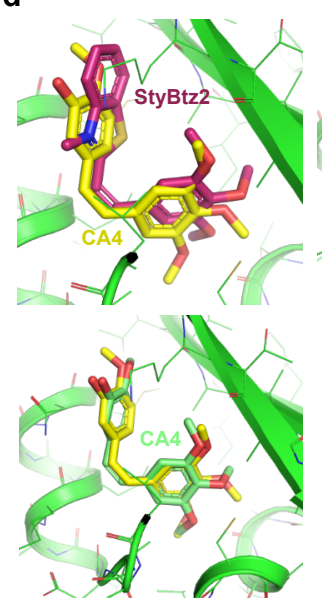

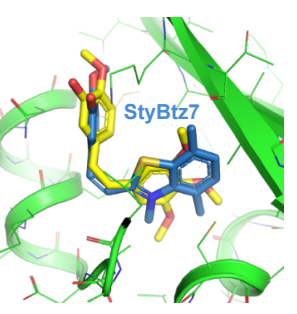

Gold Fitness Score:

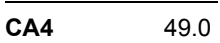

StyBtz2 55.1

StyBtz3 $\quad 47.5$

StyBtz7 $\quad 56.3$

StyTz2 51.3

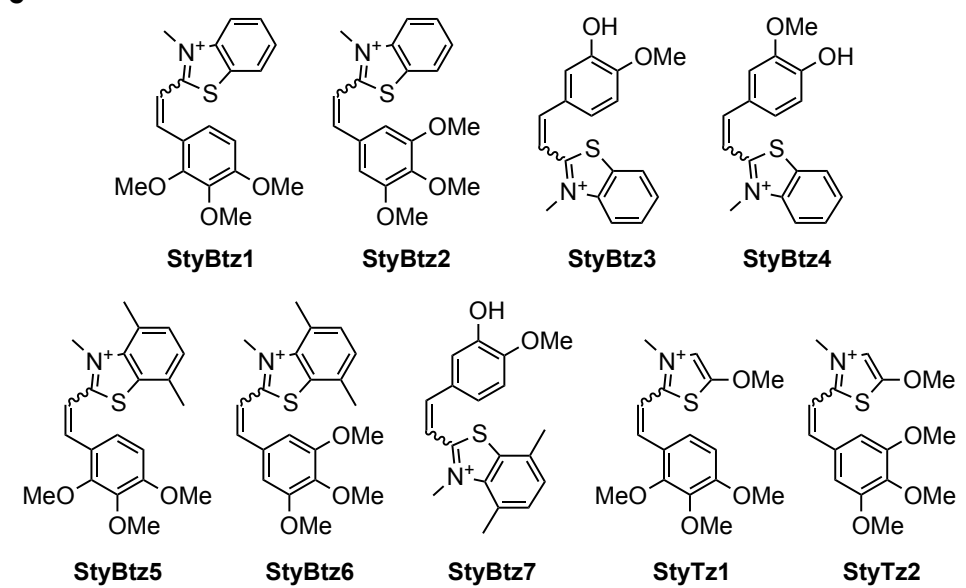

Figure 1. Design of styryl[benzo]thiazolium photopharmaceuticals. (a) The cis-active non-photoswitchable colchicine domain inhibitor CA4 has inspired photoswitchable azobenzene-based PST and SBT-based SBTub antimitotics. (b) Z-SBTub3 inhibits tubulin polymerisation and MT-dependent processes after $E \rightarrow Z$ photoisomerization with $405 \mathrm{~nm}$ light. However, its spontaneous $Z \rightarrow E$ isomerization at physiological conditions is much slower than relevant biological time-scales, making SBTubs effectively unidirectional photoswitches for cell biology. (c) Representative synthesis of styrylbenzothiazolium [StyBtz] photopharmaceutical candidate StyBtz3. (d) Docking study of StyBtz2 (red), StyBtz7 (blue) and CA4 (green) in the $\beta$-tubulin colchicine binding pocket, based on the tubulin-CA4 crystal structure (PDB: 5LYJ18). (e) StyBtz/StyTz made in this study.

Several photoswitchable tubulin inhibitors allowing spatiotemporal control over microtubule (MT) dynamics and network architecture have been developed. ${ }^{4}$ The most biologically-used are Z-active colchicine-site reagents inspired by $Z$-active stilbene combretastatin A4 (CA4) ${ }^{19}$ : PSTs $^{20}$ and SBTubs $^{21}$ (Fig 1a). Azobenzene PSTs allow bidirectional photoswitching of MT dynamics in systems up to early embryos $(E \rightarrow Z$ 
at $405 \mathrm{~nm}, Z \rightarrow E$ at $514 \mathrm{~nm}),{ }^{22,23}$ but their $Z$-isomers are rather rapidly metabolised by cellular thiol machinery preventing later-stage in vivo uses ${ }^{21}$. Heterostilbene SBTubs have better $E \rightarrow Z$ photoswitching at $405 \mathrm{~nm}$, are optically transparent above $480 \mathrm{~nm}$ to allow GFP-imaging, and are metabolically robust, so they have succeeded in various in vivo applications ${ }^{15}$ (Fig $\mathbf{1 b}$ ). However, since their $Z \rightarrow E$ relaxation is slow and they do not permit bulk $Z \rightarrow E$ photoswitching, experiments rely on diffusion to restore protein activity after $E \rightarrow Z$ photoswitching in targeted areas (see Supporting Note 1).

We reasoned that "push-pull" $\mathrm{N}$-methylated SBT analogues - styrylbenzothiazolium (StyBtz) ${ }^{24}$ and styrylthiazolium $\left(S t y T z{ }^{25}\right.$ ) - might be still better photoswitches for biological applications to MT photocontrol. Compared to heterostilbenes, we expected faster $Z \rightarrow E$ relaxation due to polarisation. Compared to both azobenzenes and heterostilbenes, we expected better solubility due to their charge, and a bathochromicallyshifted $\pi \rightarrow \pi^{*}$ band (again due to polarisation) for more efficient response to 405 or $440 \mathrm{~nm}$ lasers, giving better in vivo compatibility: while they might still be optically transparent to GFP/YFP imaging. Relaxation is the most important of these: combining $E \rightarrow Z$ photoswitching with biologically fast $Z \rightarrow E$ relaxation would allow StyBtz/StyTz to be patterned into specified $E / Z$-isomer distributions, with the biologically appropriate spatiotemporal resolution needed for MT studies. ${ }^{26}$ Although neither StyBtz nor StyTz have been tested in photopharmacology even in cell-free assays, StyBtz photochemistry has been studied: they photoisomerise reversibly in organic and aqueous media, ${ }^{27}$ and have fluorescence emission around $500 \mathrm{~nm}^{28}$ (note the similarity of push-pull StyBtz to truncated Cy dyes).

We therefore determined to design StyBtz- and StyTz-based photopharmaceuticals informed by the structure-activity relationship (SAR) known to drive tubulin binding at the colchicine binding site; ;5,29 $^{15}$ synthesise and photocharacterise them to evaluate the scaffolds' general potential as photoswitches for photopharmacology; and also to biologically evaluate these specific derivatives in cellular studies, as tubulin inhibitor photopharmaceutical candidates.

\section{Results}

\section{SAR/modelling-based design}

We designed a series of StyBtz/StyTz informed by molecular modelling and by known SAR ${ }^{29}$. We aimed both at SAR-compatible inhibitors, and at SAR-clashing regioisomeric compounds that would serve as controls for the desired mechanism of action.

We designed StyBtz1-4 (Fig 1e) as N-methylated analogues of known "parent" photoswitches SBTub1-421, with StyBtz5-7 exploring additional bulk and StyTz1-2 exploring reduced bulk. In brief, we expected that the StyBtz/StyTz might inherit the tubulin-binding performance of their parent compounds, such that in their $Z$ isomers, StyBtz2/3 and StyTz2 might be tubulin binders, whereas regioisomers StyBtz1/4 and StyTz1, as well as SAR-clashing StyBtz5 (that are likely to have similar photochemistry and off-target profiles) ought not be tubulin binders but might serve as controls for confounding off-target effects in assays (Supporting Note 2). It was not possible to guess whether StyBtz6/7 would be tolerated (Fig 1e).

Studies docking these StyBtz/StyTz in the $\beta$-tubulin colchicine binding pocket were then performed using the experimental tubulin-CA4 complex structure (PDB 5LYJ ${ }^{18} ; 2.4$ Å resolution). After re-docking of CA4 into the vacant site confirmed accurate modelling reproduction of the experimental structure (Fig 1d bottom), we docked the StyBtz / StyTz to compare their Gold ${ }^{30}$ fitness scores (Fig 1d; higher score indicates better fit). The high scores for Z-StyBtz2 (Fig 1d upper left), Z-StyBtz3, and Z-StyTz2 mirrored biological results from the neutral parents ${ }^{21}$. Bismethylated StyBtz7 (Fig 1d upper right) had the highest score of all; by comparison to StyBtz3, its extra methyl groups occupy similar positions as the methoxy carbons in prototypical colchicine site binders such as CA4 or colchicine. We now set out to test whether these compounds in particular would prove to be $Z$-specific tubulin inhibitors.

\section{Synthesis and photocharacterisation}

Synthesis of StyBtz and StyTz proceeded by aldol condensation of 2,3-dimethylbenzothiazolium salts and benzaldehydes (Fig 1c), or by $N$-alkylation of pre-assembled SBTs (ca. $40-80 \%$ yields for StyBtz and ca. $20 \%$ yields for StyTz; see Supporting Information).

StyBtz/StyTz can be $E \rightarrow Z$ photoswitched at $450 \mathrm{~nm}$ to give $E: Z$ ratios ca. $1: 9$ at the photostationary state (PSS). Unlike neutral SBTs, this PSS can be partially photoswitched back with cellularly-tolerated low intensity light, giving ca. 3:7 E:Z under $360 \mathrm{~nm}$ (Fig 2a-b; Fig S1; Fig S7). Compared to the neutral parent SBT/ST, their absorption bands are red-shifted by $\sim 40 \mathrm{~nm}$, absorbing strongly in the violet (Fig S2). 
<smiles>COc1cc(/C=C/c2sc3ccccc3[n+]2C)cc(OC)c1OC</smiles>

E-StyBtz2

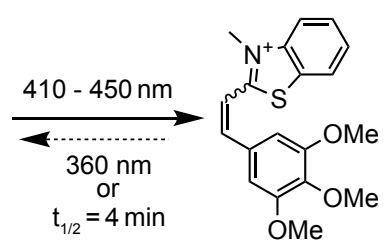

Z-StyBtz2 b

Photoequilibria StyBtz2

(PBS, 10\% DMSO)

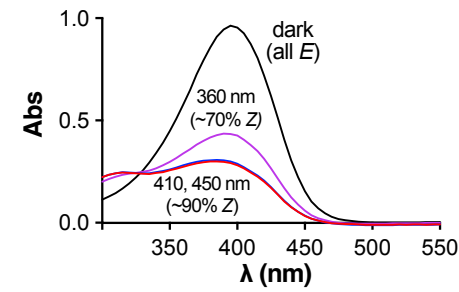

d Repeated $E \rightarrow Z$ photoisomerizations

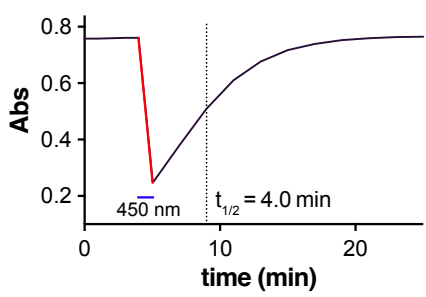
and spontaneous $Z \rightarrow E$ relaxation $\left(\mathrm{t}_{1 / 2} \sim 30 \mathrm{~s}, 37^{\circ} \mathrm{C}, \mathrm{PBS}, 10 \%\right.$ DMSO)

e

Fluorescence ex/em (PBS, 10\% DMSO)

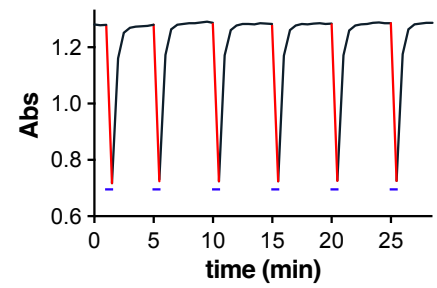

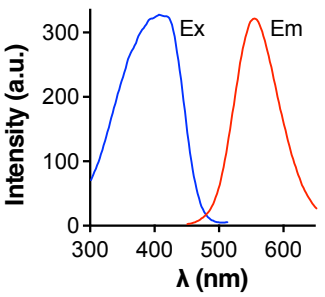

Figure 2. StyBtz photochemistry. (a-c) StyBtz2 undergoes substantial $E \rightarrow Z$ photoisomerization by blue light $(410-450 \mathrm{~nm})$ that can be partially counteracted by near-UV light $(360 \mathrm{~nm})$, and undergoes full $Z \rightarrow E$ relaxation spontaneously $\left(\mathrm{t}_{1 / 2}\right.$ at $22^{\circ} \mathrm{C}$, pH 7) within minutes (PBS $+10 \%$ DMSO). (d) Under physiological conditions, StyBtz2 can be quickly and repeatedly photoisomerized to a majority- $Z$ population before thermally relaxing to an all- $E$ state $\left(37^{\circ} \mathrm{C}, \mathrm{pH}\right.$ ) . (e) Fluorescence excitation/emission of StyBtz2 (em. $550 \mathrm{~nm} / \mathrm{ex} .450 \mathrm{~nm}$; PBS + 10\% DMSO).

Spontaneous $Z \rightarrow E$ relaxation of StyBtz restores them to all- $E$ populations with halflives of $4-90$ min at $22^{\circ} \mathrm{C}$ in $\mathrm{pH}=7$ aqueous buffer (Fig 2c; Fig S3); these rates are ca. 8-fold faster at $37^{\circ} \mathrm{C}$, enabling repeated cycles of $E \rightarrow Z$ photoisomerisation then $Z \rightarrow E$ relaxation under physiological conditions (Fig $2 \mathbf{d}$; note that $E \rightarrow Z$ isomerisation is done at low flux, as would be used in long-term cell culture assays). Surprisingly to us, at room temperature we observed insignificant $Z \rightarrow E$ relaxation of StyTz after hours (Fig S3). Spontaneous $Z \rightarrow E$ relaxation of StyBtz was strongly $\mathrm{pH}$-dependent: fast at $\mathrm{pH}$ 9, moderate at $\mathrm{pH}$, and blocked at $\mathrm{pH} 5$ (Fig S4). Relaxation was also solvent-dependent, with low-polarity EtOAc blocking relaxation, while rates in polar and/or protic DMSO, EtOH, and MeCN were comparable to those in water (Fig S5).

The fluorescence of StyBtz could be advantageous as a readout for their cellular distribution. "Good" cellular availability is vital for reagent activity, but most cellularly-used photoswitches have fluorescence brightness that is far too low for imaging readout. Matching literature expectations ${ }^{31}$, StyBtz were indeed fluorescent with ca. $100 \mathrm{~nm}$ Stokes shift (ex/em maxima typically 420/520 nm; Fig 2e, Fig S6). This large Stokes shift would later prove useful in low-background imaging of their cellular distribution.

\section{Z-StyBtz2 is cytotoxic but does not act through tubulin}

Tubulin inhibitors have antiproliferative cellular activity, due to their disruption of cell division. Accordingly, we tested the antiproliferative activity of all compounds in HeLa cervical cancer cell line, under dark (all- $E$ ) and pulse-lit ${ }^{20}$ (450 nm pulses; mostly- $Z$ ) conditions over $48 \mathrm{~h}$, hoping to see lit-specific antiproliferative effects matching our SAR/modelling predictions across the series. All StyBtz were somewhat toxic in both isomers above $20 \mu \mathrm{M}$, matching typical curveshapes for non-specific compound aggregation/precipitationbased toxicity. Only the predicted-Z-active StyBtz2 displayed appreciable light-dependent cytotoxicity with a sigmoidal dose-response curveshape as would be expected from specific activity (lit IC $\mathrm{C}_{50} \sim 6.5 \mu \mathrm{M}$, dark $\mathrm{IC}_{50}$ $\sim 66 \mu \mathrm{M})$ (Fig 3a). StyTz showed no toxicity under lit or dark regimes (Fig S9).

We therefore focused on StyBtz2 for all further investigations. We first performed immunofluorescence staining of microtubules in cells treated for $20 \mathrm{~h}$, to study the integrity of MT architecture. Matching hopes, cells treated with StyBtz2 under lit conditions showed disrupted MTs, curved and aggregating in an orbital pattern around the cell nucleus (Fig 3b) instead of the long and straight MTs of healthy cells, seen in the cosolvent controls as well as with StyBtz2 in the dark (controls and concentration scan in Fig S10).

If MT inhibition is the cause of antiproliferative cellular effects, cells should accumulate into the G2/M phase of the cell cycle, due to mitotic checkpoint failure (this is a hallmark of tubulin inhibitors such as nocodazole). We therefore used flow cytometry to analyse cell cycle after treatment, to probe the mechanism of StyBtz activity. However, neither StyBtz2 nor any other tested StyBtz disrupted cell cycle repartitioning under lit or 
dark conditions (Fig 3c, Fig S11). We concluded that, against our hopes, StyBtz2's light-dependent cellular bioactivity was not due to tubulin inhibition.

However, to understand whether StyBtz/StyTz may yet succeed as scaffolds for photopharmacology against other targets, we continued investigating Z-StyBtz2's observed bioactivity.

a

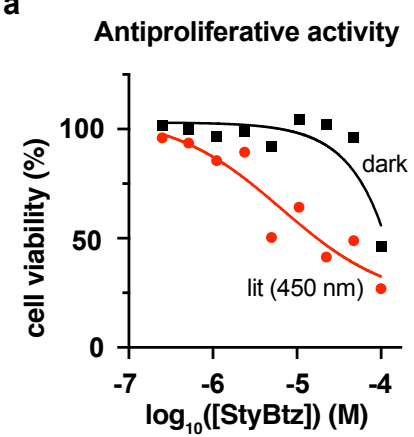

d

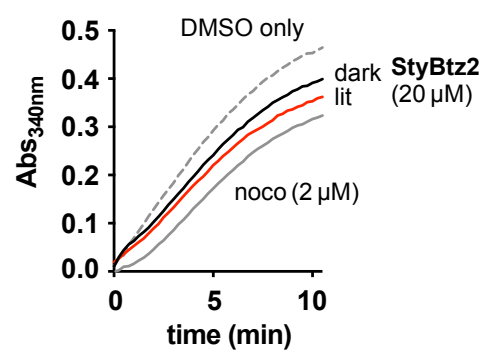

b

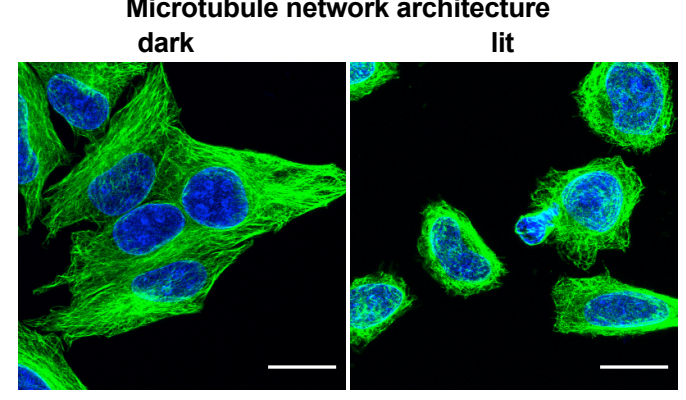

e Photosensitization assay (DPBF)

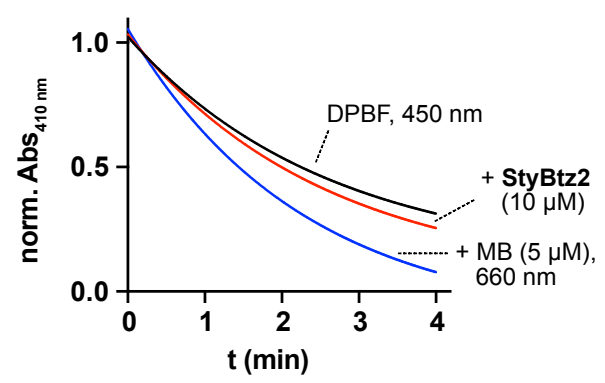
cell cycle analysis

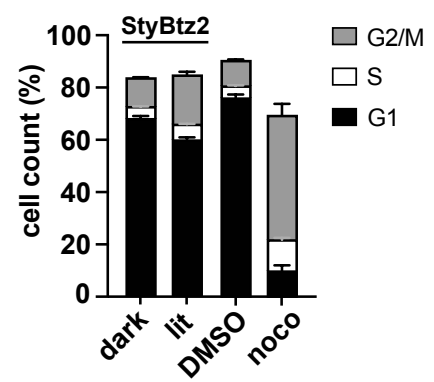

f Degradation of StyBtz2 $(25 \mu \mathrm{M})$ by GSH (10 mM)

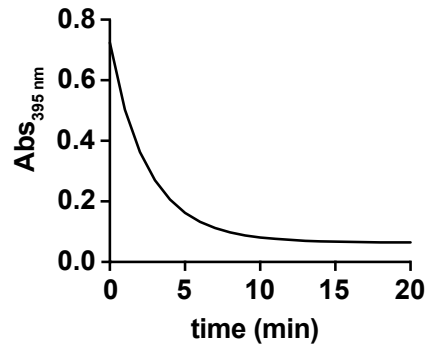

Figure 3. Mechanism of action studies. (a) StyBtz2 gives light-specific antiproliferative effects (HeLa cells, $48 \mathrm{~h}$ treatment). (b) StyBtz2 disrupts MT network architecture under $450 \mathrm{~nm}$ pulsing ("lit"), but not in the dark. (HeLa cells, $20 \mathrm{~h}$ treatment, $5 \mu \mathrm{M} ; \alpha$-tubulin in green, DNA stained with DAPI in blue, $z$-axis projection; scale bars, $20 \mu \mathrm{m}$; see Fig S10). (c) Lit StyBtz2 does not affect cell cycle repartition (HeLa cells, $24 \mathrm{~h}$ treatment, StyBtz2 at $15 \mu \mathrm{M}$ in dark or $450 \mathrm{~nm}$ pulsed conditions, nocodazole (noco; $2 \mu \mathrm{M}$ ) used as positive control for G2/M arrest; sub-G1 populations not represented; see Fig S11). (d) StyBtz2 shows no significant inhibition of cell-free tubulin polymerization in lit or dark conditions (positive control nocodazole at low concentration indicates the minimum expected threshold to assign inhibition). (e) Photobleaching of DPBF $(50 \mu \mathrm{M})$ at $450 \mathrm{~nm}$ is barely affected by StyBtz2 $(10 \mu \mathrm{M})$ but is accelerated with photosensitizers e.g. methylene blue (MB, $5 \mu \mathrm{M}$, under $660 \mathrm{~nm})$. (f) $E-$ StyBtz2 $(25 \mu \mathrm{M})$ suffers rapid degradation of the styrylbenzothiazolium chromophore by GSH (10 mM).

\section{Mechanism of action studies}

We first assayed whether StyBtz2 inhibits the polymerization of purified tubulin protein in a cell-free assay that removes the complexities of cellular localisation, metabolism, and off-targets. It had no significant inhibitory activity at high concentration $(20 \mu \mathrm{M})$ under either lit or dark conditions (Fig 3d), reinforcing that its cellular effects cannot be traced to tubulin.

Misinterpreting photosensitisation as lit-isomer toxicity is a permanent risk when working with photopharmaceuticals. ${ }^{26}$ It would be a puzzling cellular mechanism of action to propose for StyBtz2, since it is unclear why other structurally related StyBtz would not show similar activity; however, to exclude it, we tested StyBtz2 for photosensitization using the photobleaching reporter 1,3-diphenylisobenzofuran (DPBF). Its photobleaching rate was insignificantly affected by StyBtz2 $(10 \mu \mathrm{M})$, in comparison to reference photosensitizer methylene blue (MB, $5 \mu \mathrm{M}$ ) which accelerated photobleaching by $>10 \%$ (Fig 3e, Fig S8).

Photoswitch degradation by glutathione (GSH) is well attested for azobenzenes. ${ }^{21,32,33}$ Concerned that the StyBtz' polarising $\mathrm{N}$-methylation could activate the $\mathrm{C}=\mathrm{C}$ bond as a Michael acceptor electrophile, we challenged StyBtz2 (25 $\mu \mathrm{M})$ with cellularly-relevant GSH concentrations (10 mM) and monitored its integrity. StyBtz2 was rapidly degraded with a halflife under $3 \mathrm{~min}$ (Fig 3f). However, partitioning of photoswitches out of the cytosol (e.g. into lipid environments) is known to substantially impact their cellular performance ${ }^{16,34,35}$; such partitioning might shield StyBtz from GSH, enabling StyBtz2 to exert Z-specific bioactivity.

Taken together, these assays were coherent with the suggestion that Z-StyBtz2 has a light-dependent mechanism of action, not against tubulin but against other biological target/s that are potentially not cytosolic. 


\section{StyBtz localisation and activity}

We hoped that the high-Stokes-shift fluorescence of the StyBtz would allow imaging their biodistribution directly in live cells, towards identifying its targets. We first performed confocal imaging of live HeLa cells transfected with EB3-GFP, a marker for the plus tips of growing MTs which reveals them as "comets" speeding around the cell, ${ }^{36}$ after treatment with StyBtz. (Although we no longer expected StyBtz to inhibit tubulin, MT dynamics also provide a useful readout for cell shape and metabolic health). After $10 \mathrm{~min}$ incubation in the dark, we began imaging EB3-GFP comets at $488 \mathrm{~nm} / 510 \mathrm{~nm}$ excitation/emission, while imaging StyBtz2 with $405 \mathrm{~nm} / 510 \mathrm{~nm}$ (which both excites and isomerises it). StyBtz2 was initially localised into small compartments outside the nucleus, reminiscent of mitochondria; while MT dynamics were normal (Fig 4a, panel $\mathrm{t}=0 \mathrm{~s}$ ). However, within seconds of beginning imaging, StyBtz2 dispersed throughout the cell while MT comets stopped (Fig 4a, $\mathrm{t}=60 \mathrm{~s}$; Movie S1); and after minutes, cells began blebbing, detaching and dying acutely (Fig S12a).

a

StyBtz2 $(20 \mu \mathrm{M})$

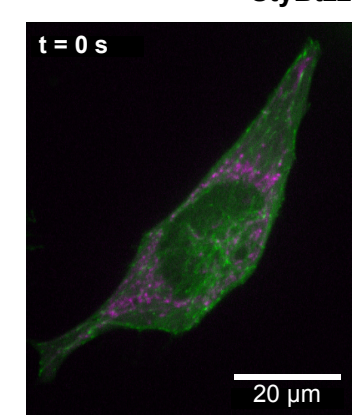

b
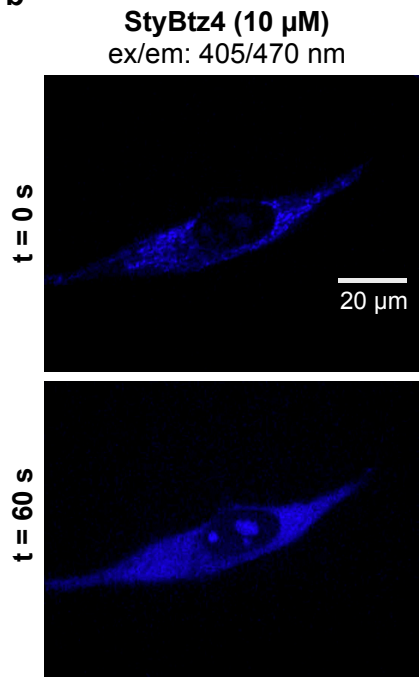

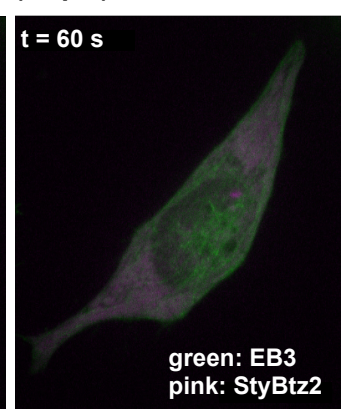

Mitochondria ex/em: $561 / 665 \mathrm{~nm}$
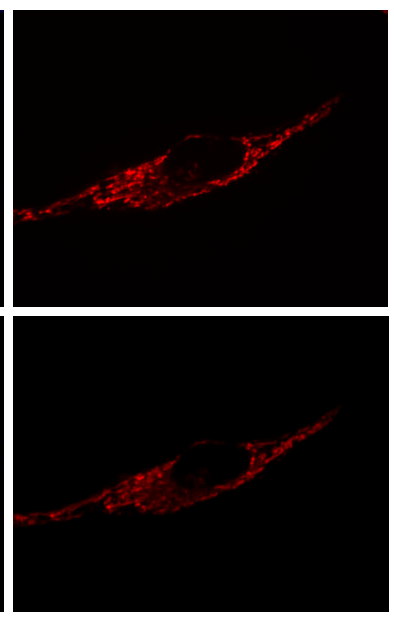

EB3 (growing MTs) ex/em: $488 / 507 \mathrm{~nm}$
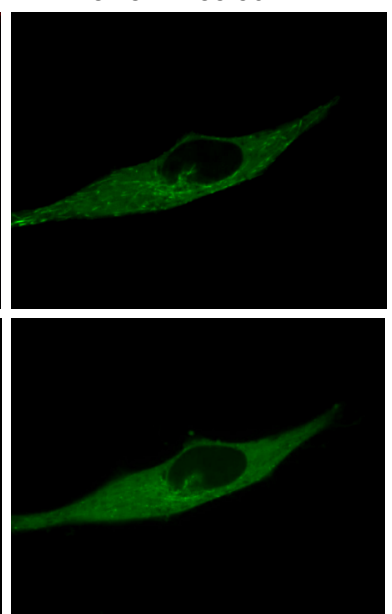

Merge
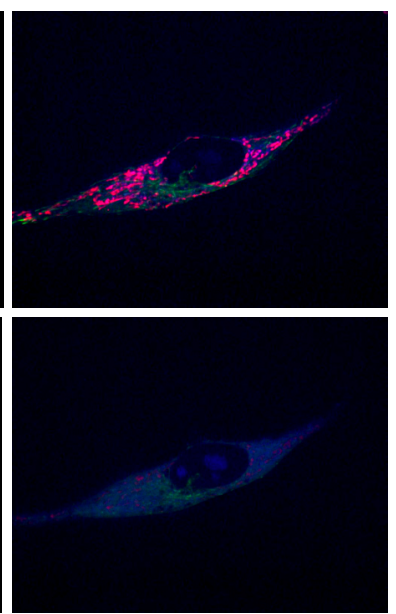

Figure 4. (a-b) HeLa cells transfected with EB3-GFP (green dots), pre-treated with StyBtz for 10 min in the dark, then imaged/photoswitched starting at time $\mathrm{t}=1 \mathrm{~s}$. (a) StyBtz2 $(20 \mu \mathrm{M}$, purple) diffuses into the cytosol after the start of $405 \mathrm{~nm}$ illuminations, while EB3 comets vanish (Movie S1). (b) StyBtz4 (blue) colocalizes with mitochondria (red) at time t=0 s. Upon photoswitching with $405 \mathrm{~nm}$ it diffuses from mitochondria to the cytosol, while EB3 comets vanish.

Since delocalized lipophilic cations (DLCs) ${ }^{37}$, which StyBtz are, often accumulate in mitochondria due to their matrix potential, ${ }^{38}$ we then co-imaged with a cationic Mitotracker dye to check if $E$-StyBtz were mitochondrially localised. Both StyBtz2 and even deprotonatable StyBtz4 colocalized with Mitotracker (Fig 4b, panels $t=0 \mathrm{~s}$ ), indicating that (a) their DLC character favours their concentration into the mitochondrial membrane, and that (b) this localisation protects them from acute chromophore biodegradation (since fluorescence is observed). Like StyBtz2, StyBtz4 was then rapidly released from mitochondria upon confocal photoactivation (Fig $\mathbf{4 b}$, $\mathrm{t}=60 \mathrm{~s}$ ), also disrupting MT dynamics (Movie S2).

We also confirmed that StyBtz offer the typically good spatiotemporal resolution of a photoresponsive tool, performing either single-cell-targeted (Fig S12b) or subcellularly-targeted (Fig S12c-d) disruption at selected times by local illumination of defined Regions of Interest (ROls). This may, for example, suit different StyBtz derivatives to applications as high-precision photoresponsive tools against mitochondrial targets. 
Taken together, we believe that the behaviour of StyBtz is consistent with: (i) accumulation into mitochondria; then (ii) under sufficiently high intensity illumination, acute depolarisation and/or rupturing of mitochondria, which is likely to be structure-dependent. ${ }^{39-41}$ Indeed, while this work was in preparation, Rivera-Fuentes reported structurally similar DLCs based on a styrylindoleninium, that likewise concentrate in mitochondria and can be released upon depolarisation, ${ }^{42}$ although photoswitching or high-intensity illumination were not examined. A partial mechanism for StyBtz bioactivity, coherent with our collected data, is given in Supporting Note 3; though further investigations are outside this report's scope of rationally designing, photocharacterising, and evaluating the as-yet unused StyBtz for a specific biological application.

\section{Conclusion}

Inspired by UV-responsive heterostilbene photoswitches that have found a range of applications for the microtubule cytoskeleton, ${ }^{15,21}$ we here examined their hitherto-untested $N$-methylated cationic analogues, StyBtz/StyTz, hoping they that could retain on-target potency as photopharmaceutical inhibitors of the cytosolic protein tubulin. We combined SAR and docking to design targets, which were then efficiently synthesised; matching the study design, we found StyBtz to have attractive photochemistry, being fasterrelaxing, better-soluble, and red-shifted photoswitches that also have self-reporting fluorescence.

While StyBtz2, which we had expected might be Z-active, did disrupt microtubule network architecture and depress cell proliferation under lit conditions, mechanistic tests of G2/M phase arrest and inhibition of purified tubulin showed that these effects cannot be traced to Z-StyBtz2 binding tubulin. Having excluded significant phototoxicity but identified problematic GSH-lability, we could use the StyBtz's self-reporting fluorescence to track their accumulation into and light-dependent release from mitochondria in acute imaging experiments. These live cell experiments also hint at mechanisms behind their light-dependent acute cytotoxicity, which can now be studied further, towards harnessing StyBtz/StyTz's mitochondrial localisation for photopharmaceutical applications against different biological targets.

\section{Author Contributions}

LG designed targets, performed synthesis, chemical analysis, photocharacterisation, cell cytotoxicity studies, and wrote the manuscript. YK performed fixed and live cell imaging. AS and LvB performed synthesis. TW performed docking studies. $\mathrm{CH}$ performed cell cytotoxicity studies and transfections. EFR performed in vitro tubulin polymerisation. JTS supervised cellular assays and analysed biological data. OTS designed the study, designed targets, supervised chemistry and wrote the manuscript.

\section{Acknowledgements}

O.T.-S. thanks the German Research Foundation (DFG: Emmy Noether grant number 400324123 ; SFB 1032 project B09 number 201269156; SFB TRR 152 project P24 number 239283807; SPP 1926 project number 426018126) and the German Ministry of Economics (BMWi: EXIST-Forschungstransfer project CytoSwitch) for funding support. J.T.-S. acknowledges support from a Joachim Herz Foundation Stipend.

\section{References}

1 K. Hüll, J. Morstein and D. Trauner, Chem Rev, 2018, 118, 10710-10747.

2 W. A. Velema, W. Szymanski and B. L. Feringa, J Am Chem Soc, 2014, 136, 2178-2191.

3 M. Borowiak, F. Küllmer, F. Gegenfurtner, S. Peil, V. Nasufovic, S. Zahler, O. Thorn-Seshold, D. Trauner and H.-D. Arndt, JACS, 2020, 142, 9240-9249.

4 O. Thorn-Seshold and J. Meiring, ChemRxiv, , DOI:10.26434/chemrxiv.14424176.v1.

5 I. Tochitsky, J. Trautman, N. Gallerani, J. G. Malis and R. H. Kramer, Scientific Reports, 2017, 7, 45487.

6 R. N. Van Gelder, Vision Research, 2015, 111, 134-141.

7 G. S. Hartley, Nature, 1937, 140, 281.

8 H. Kaufman, S. M. Vratsanos and B. F. Erlanger, Science, 1968, 162, 1487-1489.

9 H. A. Lester, M. E. Krouse, M. M. Nass, N. H. Wassermann and B. F. Erlanger, Journal Of General Physiology, 1980, 75, 207-232.

10 G. S. Kumar and D. C. Neckers, Chemical Reviews, 1989, 89, 1915-1925.

11 J. Broichhagen, J. A. Frank and D. Trauner, Acc Chem Res, 2015, 48, 1947-1960.

12 D. Lachmann, R. Lahmy and B. König, Eur J Org Chem, 2019, 2019, 5018-5024.

13 S. Wiedbrauk and H. Dube, Tet Lett, 2015, 56, 4266-4274.

14 M. W. H. Hoorens, M. Medved', A. D. Laurent, M. Di Donato, S. Fanetti, L. Slappendel, M. Hilbers, B. L. Feringa, W. Jan Buma and W. Szymanski, NComm, 2019, 10, 2390. 
15 L. Gao, J. C. M. Meiring, A. Varady, I. E. Ruider, C. Heise, M. Wranik, C. D. Velasco, J. A. Taylor, B. Terni, J. Standfuss, C. C. Cabernard, A. Llobet, M. O. Steinmetz, A. R. Bausch, M. Distel, J. ThornSeshold, A. Akhmanova and O. Thorn-Seshold, bioRxiv, 2021, 2021.03.26.437160.

16 A. Sailer, J. C. M. Meiring, C. Heise, L. N. Pettersson, A. Akhmanova, J. Thorn-Seshold and O. ThornSeshold, Angewandte Chemie International Edition, 2021, 60, 23695-23704.

17 D. B. Konrad, G. Savasci, L. Allmendinger, D. Trauner, C. Ochsenfeld and A. M. Ali, JACS, , DOI:10.1021/jacs.9b10430.

18 R. Gaspari, A. E. Prota, K. Bargsten, A. Cavalli and M. O. Steinmetz, Chem, 2017, 2, 102-113.

19 G. R. Pettit, S. B. Singh, E. Hamel, C. M. Lin, D. S. Alberts and D. Garcia-Kendal, Experientia, 1989, 45, 209-211.

20 M. Borowiak, W. Nahaboo, M. Reynders, K. Nekolla, P. Jalinot, J. Hasserodt, M. Rehberg, M. Delattre, S. Zahler, A. Vollmar, D. Trauner and O. Thorn-Seshold, Cell, 2015, 162, 403-411.

21 L. Gao, J. C. M. Meiring, Y. Kraus, M. Wranik, T. Weinert, S. D. Pritzl, R. Bingham, E. Ntouliou, K. I. Jansen, N. Olieric, J. Standfuss, L. C. Kapitein, T. Lohmüller, J. Ahlfeld, A. Akhmanova, M. O. Steinmetz and O. Thorn-Seshold, Cell Chemical Biology, 2021, 28, 228-241.e6.

22 J. Zenker, M. D. White, R. M. Templin, R. G. Parton, O. Thorn-Seshold, S. Bissiere and N. Plachta, Science, 2017, 357, 925-928.

23 J. Zenker, M. D. White, M. Gasnier, Y. D. Alvarez, H. Y. G. Lim, S. Bissiere, M. Biro and N. Plachta, Cell, 2018, 173, 776-791.

24 A. Gáplovský, P. Chabreček and V. Sutoris, 1988, 5.

25 A. Dondoni, G. Fantin, M. Fogagnolo, A. Medici and P. Pedrini, Tet, 1988, 44, 2021-2031.

26 O. Thorn-Seshold, in Molecular Photoswitches, Wiley, 2022.

27 P. J. Coelho, M. C. R. Castro and M. M. M. Raposo, DyePig, 2015, 117, 163-169.

28 M. M. El-Hendawy, T. A. Fayed, M. K. Awad, N. J. English, S. E. H. Etaiw and A. B. Zaki, JPP-A, 2015, 301, 20-31.

29 G. C. Tron, T. Pirali, G. Sorba, F. Pagliai, S. Busacca and A. A. Genazzani, J Med Chem, 2006, 49, 30333044.

30 G. Jones, P. Willett, R. C. Glen, A. R. Leach and R. Taylor, Journal Of Molecular Biology, 1997, 267, 727748.

31 R. Krieg and K.-J. Halbhuber, Journal Of Molecular Histology, 2004, 35, 471-487.

32 J. E. Sheldon, M. M. Dcona, C. E. Lyons, J. C. Hackett and M. C. T. Hartman, Org Biomol Chem, 2016, 14, 40-49.

33 Y. An, C. Chen, J. Zhu, P. Dwivedi, Y. Zhao and Z. Wang, Frontiers Of Chemical Science Engineering, 2020, 14, 880-888.

34 A. Sailer, F. Ermer, Y. Kraus, R. Bingham, F. H. Lutter, J. Ahlfeld and O. Thorn-Seshold, BJOC, 2020, 16, 125-134.

35 M. Müller, K. Niemeyer, N. Urban, N. K. Ojha, F. Zufall, T. Leinders-Zufall, M. Schaefer and O. ThornSeshold, ChemRxiv, , DOI:10.26434/chemrxiv-2022-hvh6b.

36 L. Gao, J. C. M. Meiring, C. Heise, A. Rai, A. Müller-Deku, A. Akhmanova, J. Thorn-Seshold and O. ThornSeshold, Angewandte Chemie International Edition, 2021, n/a, e202114614.

37 V. R. Fantin, M. J. Berardi, L. Scorrano, S. J. Korsmeyer and P. Leder, Cancer Cell, 2002, 2, $29-42$.

38 W.-L. Wu, X. Zhao, L.-L. Xi, M.-F. Huang, W.-H. Zeng, J.-Y. Miao and B.-X. Zhao, Anal Chim Acta, 2017, 950, 178-183.

39 J. Hüser, C. E. Rechenmacher and L. A. Blatter, Biophys J, 1998, 74, 2129-2137.

40 J. A. Dykens and A. K. Stout, in Methods in Cell Biology, Academic Press, 2001, vol. 65, pp. $285-309$.

41 I. Belostotsky, S. da Silva, M. Paez and G. Indig, Biotechnic Histochemistry, 2011, 86, 302-314.

42 J. Nguyen, A. Tirla and P. Rivera-Fuentes, OBC, 2021, 19, 2681-2687. 